\title{
Observations of microwave bursts with different types of fine structure using data with high spatial and spectral resolution
}

\author{
A. T. Altyntsev ${ }^{1}$, N. N. Kardapolova ${ }^{1}$, A. A. Kuznetsov ${ }^{1}$, \\ S. V. Lesovoi ${ }^{1}$, N. S. Meshalkina ${ }^{1}$, R. A. $\operatorname{Sych}^{1}$, Y. Yan ${ }^{2}$ \\ ${ }^{1}$ Institute of Solar-Terrestrial Physics SB RAS, Irkutsk, Russia, email: altyntsev@iszf.irk.ru \\ ${ }^{2}$ National Astronomical Observatories, Beijing, China, email: yyh@bao.ac.cn
}

\begin{abstract}
The observations of the bursts with fine structures are one of the best remote sensing probe of the primary energy release sites in solar flares. The Siberian Solar Radiotelescope (SSRT) gives us the unique possibility of direct measurements of their positions with temporal resolution up to $14 \mathrm{~ms}$ and spatial resolution of a few arcsec. National Astronomical Observatories of China (NAOC) spectropolarimeters $(5.2-7.7 \mathrm{GHz})$ provide us with the simultaneous spectral data around the SSRT receiving frequency $(5.7 \mathrm{GHz})$. An analysis is made of the subsecond pulses observations: short wide range pulses, U-type microwave bursts, the bursts with zebra pattern.
\end{abstract}

The solar radio and microwave bursts with fine temporal and spectral structure are one of the most important sources of information about processes in the solar corona. SSRT provides high spatial and temporal resolution at the frequency $5.7 \mathrm{GHz}$. The NAOC spectropolarimeters provide high temporal and spectral resolution in wide frequency range (2.6-3.8 and $5.2-7.6 \mathrm{GHz})$. These unique combined data provide us by the relatively complete information about the sources of microwave emission.

Microwave bursts show a high variety of types. U-type bursts are the bursts with complex frequency drift, in which the emission frequency firstly decreases, and then increases again. As the observations by SSRT have shown, the source displacement during the interval between the U-burst branches does not exceed 3", or about 3 thousand km. Therefore the observed U-type bursts cannot be explained by an emission of an electron beam traveling in a closed magnetic structure. In contrary we argue that microwave Ubursts are emitted by compact sources, and the frequency drift of emission is caused by density variation due to fast local heating of plasma.

Zebra patterns consist of a number of parallel bright stripes. Such structures are quit common in metric and decimetric bursts, but at $5.7 \mathrm{GHz}$ the zebra pattern was observed at the first time. A frequency interval between stripes was $0.16 \mathrm{GHz}$, that corresponds to magnetic field about $60 \mathrm{G}$. The source size of zebra pattern does not exceed 10", and the sources of different stripes coincide. The polarization degree of emission was as high as $100 \%$; the comparison with the magnetogram has shown that the emission corresponds to extraordinary wave. Our opinion is that the most probable mechanism of zebra pattern emission is nonlinear coupling of harmonics of Bernstein waves with numbers about $17-18$.

Statistical analysis of bursts with fine structure has shown that the emission sources are often situated at the distances less than 10" from the magnetic neutral line (apparently, in the tops of the magnetic loops). In other cases polarization of subsecond pulses corresponds, as a rule, to the ordinary wave, whereas the polarization of background burst most often corresponds to extraordinary wave. 

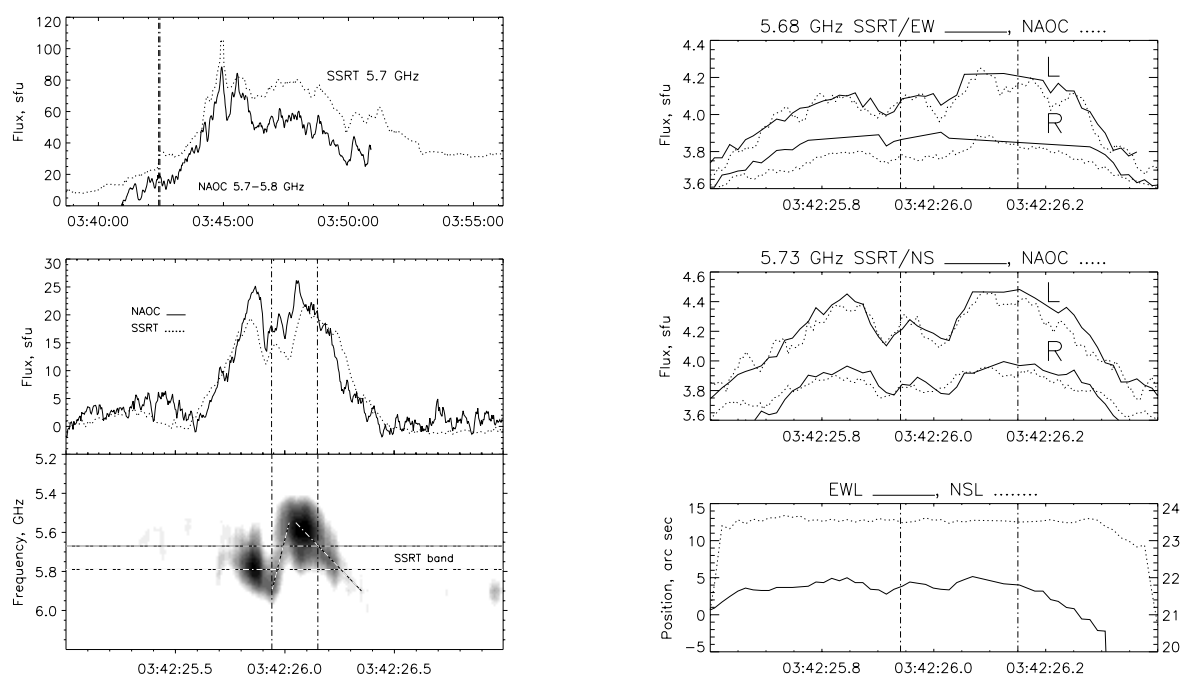

Figure 1. Dynamic spectra and time profiles of the burst with frequency drift. Darker areas correspond to higher emission intensity. One-dimensional coordinates of the burst source are plotted at the right bottom panel.
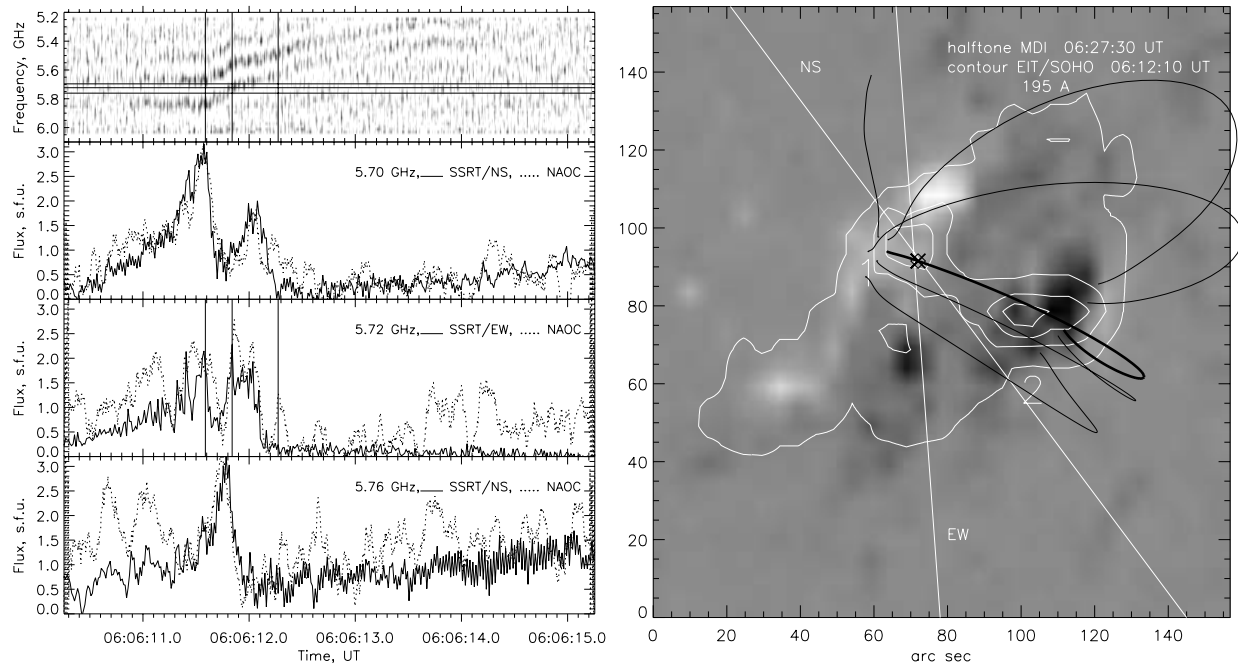

Figure 2. Dynamic spectra and time profiles of the burst with zebra pattern (left). Structure of the corresponding active region with the zebra pattern source shown (right).

The microwave bursts of subsecond duration can be divided into two groups. Most of such bursts have polarization opposite to the polarization of the background (more smooth) microwave burst; the sources of the subsecond pulses of this type are shifted from the sources of the corresponding background bursts in horizontal direction. Some subsecond pulses has the same polarization as the background microwave burst, their sources are shifted from the sources of the corresponding background bursts in vertical direction.

\section{Acknowledgements}

This work was supported by the RFBR (grant numbers 02-02-39030 and 03-02-16229) and by the Russia Department of education (grant number E02-3.2-489). 